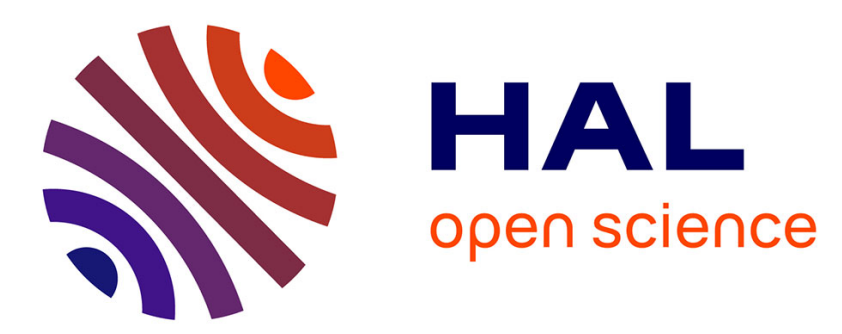

\title{
Quand recherche et pédagogie se croisent : étudier les chemins de l'école à Maripasoula en Guyane française
}

\author{
Alexandra Vié
}

\section{To cite this version:}

Alexandra Vié. Quand recherche et pédagogie se croisent : étudier les chemins de l'école à Maripasoula en Guyane française. La nouvelle revue - Éducation et société inclusives, 2018. hal-03237434

\section{HAL Id: hal-03237434 \\ https://hal-inshea.archives-ouvertes.fr/hal-03237434}

Submitted on 26 May 2021

HAL is a multi-disciplinary open access archive for the deposit and dissemination of scientific research documents, whether they are published or not. The documents may come from teaching and research institutions in France or abroad, or from public or private research centers.
L'archive ouverte pluridisciplinaire $\mathbf{H A L}$, est destinée au dépôt et à la diffusion de documents scientifiques de niveau recherche, publiés ou non, émanant des établissements d'enseignement et de recherche français ou étrangers, des laboratoires publics ou privés. 


\title{
QUAND RECHERCHE ET PÉDAGOGIE SE CROISENT : ÉTUDIER LES CHEMINS DE L'ÉCOLE À MARIPASOULA EN GUYANE FRANÇAISE
}

\author{
Alexandra Vié
}

I.N.S.H.E.A. | « La nouvelle revue - Éducation et société inclusives »

2018/3 N83-84 | pages 165 à 182

ISSN 2609-5211

Article disponible en ligne à l'adresse :

https://www.cairn.info/revue-la-nouvelle-revue-education-et-societeinclusives-2018-3-page-165.htm

Distribution électronique Cairn.info pour I.N.S.H.E.A..

(C) I.N.S.H.E.A.. Tous droits réservés pour tous pays.

La reproduction ou représentation de cet article, notamment par photocopie, n'est autorisée que dans les limites des conditions générales d'utilisation du site ou, le cas échéant, des conditions générales de la licence souscrite par votre établissement. Toute autre reproduction ou représentation, en tout ou partie, sous quelque forme et de quelque manière que ce soit, est interdite sauf accord préalable et écrit de l'éditeur, en dehors des cas prévus par la législation en vigueur en France. Il est précisé que son stockage dans une base de données est également interdit. 


\title{
Quand recherche et pédagogie se croisent : étudier les chemins de l'école à Maripasoula en Guyane française
}

\author{
Alexandra VIÉ \\ Doctorante au Grhapes - INSHEA \\ ED 139 - Université Paris Nanterre
}

Résumé: Cet article vise à interroger l'expérience scolaire des élèves inscrits en UPE2A, mais également les pratiques enseignantes dans une école élémentaire du bourg de Maripasoula en Guyane française. Nous axerons nos propos sur un travail collaboratif au cours duquel praticiens et chercheur se sont intéressés aux chemins que les enfants empruntent tous les jours pour se rendre à l'école. II est donc question d'exposer la méthodologie créée dans ce triptyque d'acteurs, mais aussi de mettre en avant les enjeux que représentent les transports scolaires dans l'accès à la scolarisation. Dès lors, les projets scolaires se doivent d'être pensés à la lumière de I'hypermobilité des familles de Maripasoula, souvent liée à une forte précarité.

Mots-clés: Formation - Frontières - Guyane française - Migrations - Scolarisation - Transports scolaires.

When research and pedagogy intersect : studying the paths of the school in Maripasoula in French Guiana

Summary: This article aims to question the school experience of children enrolled in UPE2A but also teaching practice in an elementary school in the town of Maripasoula, French Guiana. We will focus on a collaborative work in which practitioners and researcher are interested in the paths that children take every day to get to school. It is therefore at once a question of presenting the methodology created in this triptych of actors. This paper wants also analyse the challenges that school transport represents in the access to education as well as the high level of mobility of the families of Maripasoula, often related to a high degree of precariousness.

Keywords: Borders - French Guiana - Migration - Schooling - School transport - Training. 
Dans la plus grande commune ${ }^{1}$ de France, les seuls transports scolaires publics sont des transports fluviaux. Située au sud-ouest du territoire de la Guyane, Maripasoula est frontalière du Suriname et du Brésil. Les fleuves séparent cette terre d'Amazonie française de ses voisins. Dans cette commune de près de onze mille habitants, la population y est très inégalement répartie, entre un bourg densément peuplé et des villages ruraux ${ }^{2}$ situés à plusieurs heures de navigation. À l'instar d'autres communes de Guyane, tous les matins, un ballet de pirogues s'anime sur le fleuve, passant de village en village pour récupérer les enfants et les conduire dans les onze établissements scolaires ${ }^{3}$ recensés dans la commune. Le bourg ne fait pas exception. Une pirogue permet aux enfants, éloignés par la route ou quand cette dernière devient impraticable, de venir à l'école. En effet, dans ce bourg d'environ neuf mille habitants et de mille six cents élèves ${ }^{4}$, il n'existe pas de cars scolaires. Les plus jeunes, scolarisés en maternelle, sont transportés en moto ou taxis collectifs. Les autres viennent à pied. Cependant, tous les enfants scolarisés n'habitent pas dans le bourg de la commune ou dans les villages français avoisinants. Certains vivent de I'autre côté du fleuve Maroni, au Suriname et sont scolarisés en Guyane française. Dans le cadre de cet article, nous nous intéressons à l'expérience scolaire d'enfants inscrits dans l'une des deux écoles élémentaires du bourg de la commune. Notre réflexion s'inscrivant dans une recherche ${ }^{5}$ sur l'accueil et l'inclusion des Enfants allophones nouvellement arrivés (EANA), nous avons choisi cette école, dans la mesure où elle est la seule de la commune à bénéficier d'une UPE2A ${ }^{6}$. Dans la circulaire de cadrage, ce dispositif est destiné aux enfants allophones nouvellement arrivés en France ou dans le système éducatif français. Or, en Guyane française, les UPE2A du premier degré accueillent aussi des enfants allophones nés et scolarisés sur le territoire. Une partie de ce projet de recherche est consacrée au ressenti des élèves quant à leur place dans l'école. C'est dans cette perspective que nous avons décidé de nous intéresser aux chemins que les enfants empruntent tous les jours pour se rendre à l'école. En effet, les premiers constats en matière d'absentéisme, de pratique intermittente de l'école (Missaoui, 2007) et de précarité socio-économique des familles, nous ont conduits à interroger les projets à l'œuvre dans le fait d'aller à l'école. Quels sont les projets familiaux et individuels qui sous-tendent le fait de se rendre dans un établissement scolaire? Quels sont les trajets que parcourent

1. La commune s'étend sur $18761 \mathrm{~km}^{2}$.

2. Maripasoula est constituée d'un vaste bourg ainsi que d'une multitude de villages implantés en bordure de rivière dont les principaux: New Wacapou en aval du bourg, Alö̈ké et Tédamali-Boussoussa, tous deux en amont immédiat du bourg et plus au Sud de la commune, Elaé, Kayodé, Twenké, Taluen, Antecume Pata et Pidima constituant les villages du " pays indien ", Rapport du plan local d'urbanisme, 2013, p. 6.

3. Cinq établissements sont situés dans le bourg, dont le collège et six établissements du premier degré sont situés dans les villages (New Wacapou, Elahé, Cayodé, Taluen, Antecume Pata, Pidima).

4. Chiffres 2016-2017. Pour le premier degré: affaires scolaires de Maripaosula, pour le second degré: rectorat de Guyane. Constat d'effectifs d'élèves du second degré; Secteurs public et privé sous contrat en Guyane.

5. Projet de recherche: "Accueillir, scolariser et accompagner les élèves primo arrivants, itinérants ou de famille du voyage. Élèves et professionnels du champ éducatif face aux enjeux de l'inclusion "; Educinclu, INSHEA, Suresnes. Ce projet comprend trois terrains de recherche: Bordeaux, Versailles et la Guyane française.

6. UPE2A: Unité pédagogique pour élèves allophones arrivants, créée par la circulaire du ministère de l'Éducation nationale n²012-141 du 2 octobre 2012 intitulée "Organisation de la scolarité des élèves allophones nouvellement arrivés ". 


\section{POLITIOQUES INCLUSIVES D’ÉDUCATION}

chaque jour les enfants? Quelle est l'organisation familiale, communautaire ou institutionnelle nécessaire pour rejoindre sa classe ? Voici un ensemble d'interrogations qui nourrissent notre recherche et pour lequel plusieurs investigations de terrain ont été réalisées. Par cet article, nous souhaitons d'une part, interroger le passage entre le monde scolaire et l'environnement familial et d'autre part, mettre en valeur un travail méthodologique construit conjointement entre chercheur et praticiens?. Ainsi, dans une première partie, nous partagerons nos réflexions méthodologiques en insistant sur la construction d'outils de recherche ayant des apports pédagogiques. Dans une deuxième partie dédiée aux chemins à défricher, nous présenterons les premiers résultats liés aux trajets scolaires et à l'environnement familial. Enfin, dans une dernière partie, nous réfléchirons à l'apport de l'alliance recherche-pédagogie dans la formation continue des enseignants du premier degré en territoire enclavé.

\section{À LA CROISÉE DES MÉTHODOLOGIES...}

\section{La démarche de recherche : une construction au fil des terrains}

Afin d'interroger l'expérience scolaire des élèves, nous avons développé une méthodologie prenant appui sur différentes approches collaboratives (Armagnague et al., 2017; Farmer, 2016; Hubert, 2014). Ces auteurs travaillant tous auprès d'élèves migrants ont tenté de recueillir leur point de vue par des méthodes participatives s'exprimant à travers des conceptions artistiques, de la réalisation d'autoportraits ou encore de l'écriture de fiction. Investiguant dans I'UPE2A de Maripasoula depuis la rentrée scolaire 2016, nous avons pu étayer notre méthodologie d'une année sur l'autre.

Un premier recueil de données a eu lieu durant l'année scolaire 2016-2017. Au cours de différentes séances en classe, nous avons questionné l'environnement familial des enfants. Avec l'enseignante de I'UPE2A, nous leur avons demandé individuellement de représenter leur habitat et leur famille. Après une phase d'autonomie, nous sommes passées auprès de chacun d'entre eux pour qu'ils puissent s'exprimer sur les membres de la famille qu'ils avaient choisi de présenter. Nous avons choisi le dessin réflexif dans la mesure où il diminue l'influence du chercheur en offrant un espace d'échange moins directif (Farmer, 2016, p. 109). Pour ce faire, ils avaient à leur disposition, sur une feuille, le contour de deux maisons vides et des petits personnages à coller ou à dessiner. Du fait de leur inscription en UPE2A, nous avons estimé qu'une partie des enfants étaient migrants. Ne dessiner qu'un seul lieu masquait leur parcours et mettait sous silence leur migration. Cependant, nous n'avions pas envisagé que certains enfants présenteraient plusieurs habitations toutes situées dans le bourg de la commune. À la suite de cette activité, nous avons organisé une sortie collective dans le bourg afin d'aller à la découverte de leur maison et éventuellement de leur famille. Les élèves nous ont présenté le lieu qu'ils ont associé à leur espace de vie ou celui qui avait du sens pour eux. Par exemple, une petite fille nous a emmenées

\footnotetext{
7. II s'agit de l'enseignante de I'UPE2A et un conseiller pédagogique du Casnav.

Casnav: centre académique pour la scolarisation des Élèves allophones nouvellement arrivés (EANA) et des Enfants issus de familles itinérantes et de voyageurs (Efiv).
} 
devant la maison de sa sœur ainée; une autre sur le lieu de travail de sa mère. Ils ont ensuite photographié ce lieu afin de constituer un livre numérique aujourd'hui consultable dans I'UPE2A. En 2017-2018, nous avons poursuivi la collecte de données. Cette fois-ci, nous avons pris le parti d'accompagner individuellement les enfants du portail de l'école jusqu'à leur domicile. En effet, la sortie collective réalisée un an plus tôt ne permettait pas d'appréhender les chemins parcourus par les enfants, ni même de savoir avec qui ils effectuaient le trajet quotidiennement. Le rythme scolaire de la commune étant celui de la journée continue, la sortie a lieu à 12 h 30 . Pendant plus d'une semaine, nous avons accompagné les enfants, en les invitant à photographier les éléments qu'ils considéraient comme importants sur leur parcours. Le lendemain, en classe avec l'aide de l'enseignante, chaque enfant représentait son trajet et l'expliquait aux autres.

Suite à la présentation de ces premières expérimentations aux enseignants, il est apparu essentiel d'y apporter un volet pédagogique. En effet, plusieurs remarques ont retenu notre intérêt. La première interrogeait directement le sens pédagogique du projet. Dans quelle mesure ce travail participait-il aux apprentissages? II a également été question du rôle des enseignants dans I'histoire personnelle des enfants. Dans quelle mesure la connaissance des trajets scolaires et de l'environnement familial était-elle du ressort de l'enseignant? Ces remarques nous ont conduits à affiner notre expérimentation de recherche en proposant à un conseiller pédagogique du Casnav et formateur à l'Espé ${ }^{8} d^{\prime}$ intégrer le projet pour s'enrichir de son expertise. Par cette approche collaborative entre professionnels (enseignants-formateur-chercheur) nous souhaitions favoriser un travail de recherche permettant aux enfants d'acquérir des compétences, afin de proposer ces expérimentations à d'autres enseignants. D'autre part, il s'agissait d'analyser les rapports professionnels entre enseignants et conseiller pédagogique dans un territoire isolé tel que l'est celui de Maripasoula. Différentes phrases de travail ont donc été organisées, conduisant à la réalisation d'une semaine d'expérimentation dans la classe de l'enseignante de I'UPE2A, en mai 2018.

\section{L'approche pédagogique : donner du sens}

Durant cette semaine, le conseiller pédagogique a travaillé en co-intervention avec les enfants et l'enseignante, en présentant une séquence et les démarches attendues auprès des EANA. Convaincu de l'utilité des approches plurilingues pour la valorisation des langues-cultures des élèves en Guyane, le conseiller a choisi de travailler à partir d'un album bilingue en randonnée. Nous reviendrons sur le principe de l'album en randonnée par la suite.

" Je pense que l'académie est dans une problématique plurilingue, et ma réponse à cette problématique n'est ni une réponse française langue seconde parce que toutes les familles ne sont pas dans le français langue seconde, et toutes les familles ne sont pas non plus dans du français langue étrangère. [...] Ici, la langue clairement véhiculaire, c'est plus le créole, et le français selon

8. Espé: École supérieure du professorat et de l'éducation. 


\section{POLITIOQUES INCLUSIVES D’ÉDUCATION}

les zones géographiques. Sur Saint-Laurent, on est sur du nenge tongo mais aussi du créole et y a pas un endroit où on peut dire... Moi, je me promène à Cayenne, tu descends la rue à Cayenne et t'entends sept-huit langues sur six cents mètres, donc on n'est pas dans quelque chose qui est catégorisé FLE ou FLS. " (Conseiller pédagogique Casnav, 2017).

À travers de nombreux travaux de recherche en sociolinguistique réalisés en Guyane française, Isabelle Leglise et Sophie Alby ont mis en avant le plurilinguisme des enfants - de migrants, en particulier - et des familles (Alby, 2008; Leglise, 2008). Ces derniers posséderaient dès leurs premières années de socialisation plusieurs langues dans leur répertoire, alternant les langues en fonction des situations et de leurs interlocuteurs (Leglise, 2008, p. 4). Ainsi, le conseiller pédagogique est parti d'un conte ${ }^{9}$ écrit en Guyane par les intervenants en langue maternelle ${ }^{10}$. II s'est proposé de conduire le début de la séquence pour montrer une pratique pédagogique possible. Cette démarche s'inscrit dans sa lettre de mission " proposer des ateliers de découverte et de pratiques artistiques, linguistiques ou sportives". Il a commencé par une première écoute du conte en langue maternelle, puis a demandé aux enfants de donner le nom des animaux rencontrés dans I'histoire en français ou en nenge tongo ${ }^{11}$. Ainsi par un travail de réception orale, les enfants ont pu raconter I'histoire, sans I'avoir entendue au préalable, en français. Les enfants d'origine brésilienne présents dans le groupe ont également réussi à présenter le conte, guidés par les informations du conseiller pédagogique. Lors de la deuxième séance, en binôme, les enfants ont remis en ordre les personnages rencontrés par le protagoniste de l'histoire, un chien, à l'aide d'images d'animaux sous la forme d'un chemin de l'histoire ${ }^{12}$. Lors d'une troisième séance, ils ont reconstruit l'histoire par un travail d'art plastique en confectionnant une boîte à histoires. Pour le conseiller pédagogique, ce dernier travail avait pour objectif de développer les interactions orales entre élèves, mais aussi entre les élèves et la professeure d'UPE2A. II était important pour lui d'amener l'enseignante à prendre conscience de la démarche actionnelle.

9. Conte A koy fu baa dagu - La promenade du chien, auteur/illustrateur: Ingrid Joachim, Marie-France Doudou, Alain Djani, Maryline Amayo, langue nenge tongo, publié sur le site académique langues de Guyane.

10. Le dispositif aujourd'hui appelé Intervenants en langue maternelle (ILM) est né en 1998, sous le nom de Médiateurs culturels bilingues (MCB), d'une concertation de responsables du rectorat de Guyane, de directeurs d'écoles et de linguistes du Centre d'études des langues indigènes d'Amérique (CELIA), travaillant à I'IRD (Institut de recherche pour le développement).

11. Le terme nenge tongo ou nengee revoient aux différentes variantes des langues aluku, ndjuka et paramaca. Ces langues font partie de la famille des créoles à base lexicale anglaise et sont parlées principalement par les populations Noires-Marrons. Les Marrons ou Noirs-Marrons également appelés "Bushinengues" (hommes de la forêt) sont pour la majorité des descendants d'esclaves déportés d'Afrique vers le Suriname, ancienne colonie hollandaise, qui ont fui les exploitations pendant le temps de l'esclavage. Ils sont nés des grands mouvements de marronnage. Il existe différentes ethnies en Guyane: les Saramaca, les Djuka, les Boni ou Aluku, les Parmaca. À Maripasoula, I'ethnie majoritaire est Aluku.

12. Le travail s'inspire des activités ritualisées en maternelle présentées dans - Ressources maternelle Mobiliser le langage dans toutes ses dimensions - Partie I - L'oral - Ressources pour la classe - Activités ritualisées - du ministère de l'Éducation nationale. 
"Dans son [celle de l'enseignante de I'UPE2A] approche pédagogique, on retrouve à nouveau l'héritage d'une démarche qui a longuement été utilisée en Guyane, axée autour de "langage en fête," une méthode proposée qui était une démarche communicative avec des entrées thématiques, c'est-à-dire une grosse confusion sur la manière d'aborder la langue, c'est-à-dire que les enseignants, majoritairement en UPE2A, abordent la langue par le lexique et pas par la structure. [...] Avec l'Europe et le CECRL qui est apparu dans les années 2000, on a eu un basculement vers ce qu'on appelle des approches actionnelles. » (Conseiller pédagogique Casnav, 2018).

Comme le stipule le CECRL ${ }^{13}$, dans la démarche actionnelle, l'apprenant n'effectue plus une succession d'exercices, mais une suite de tâches liées à des activités sociales, fondées sur l'interaction, immédiatement transposables en situation réelle de communication.

"Moi, je pense quand on fait des activités qui sont des activités comme on a fait ce matin [confection de la boîte à histoires] où on sort du modèle transmissif, et où on instaure une relation langagière actionnelle sur une activité, on constate, quand on interroge les enfants sur "donne-moi la boîte, amène-moi le scotch, passe-moi ci, fais ça," les enfants ils comprennent les consignes qui leur sont données. Si c'est fait en action, ils savent très bien identifier ce que c'est qu'une paire d'oreilles, une paire d'yeux, un nez, etc. donc, ils ont pas besoin de le faire de façon lexicale, c'est fait dans l'activité, et franchement, ce matin, j'ai vu peu d'élèves en difficulté sur les consignes qui leur étaient données. Moi, j'ai trouvé qu'on était dans des rapports communicatifs où y avait peu de problèmes de compréhension. " (Conseiller pédagogique Casnav, 2018).

L'autre choix pédagogique a été la sélection d'un album en randonnée ${ }^{14}$. II s'agit de récits courts à structure répétitive par accumulation, par juxtaposition. Ce type d'album permet de faire le lien entre la chaîne orale et la chaîne écrite, mais aussi de s'approprier et de mémoriser des structures, pouvant être réutilisées dans un nouveau récit. C'est sur ce modèle que les enfants ont reconstruit leur propre chemin allant de l'école au domicile et qu'ils ont replacé dans l'ordre les éléments qu'ils avaient photographiés ${ }^{15}$ sur leur trajet. Ces derniers accompagnés de l'enseignante et du conseiller pédagogique ont travaillé à l'écrit, donnant une explication à chaque élément présenté.

Par ce volet pédagogique, la recherche s'est inscrite dans le cadre scolaire donnant ainsi du sens aux différents protagonistes. Les enfants ont pu développer des compétences langagières à l'oral et à l'écrit, l'enseignante et le conseiller pédagogique ont été placés en position d'experts, au cœur de la réalisation des ateliers laissant

13. CECRL: Cadre européen commun de référence pour les langues.

14. Ces contes sont surtout utilisés dans les classes de maternelle. Le conte Roule galette de Natha Caputo et Pierre Belvès écrit en 1993 aux éditions Flammarion est un exemple de conte en randonnée.

15. Dans un souci de réalisation, 5 photographies par enfant ont été choisies pour le support final. 


\section{POLITIOQUES INCLUSIVES D'ÉDUCATION}

ainsi au chercheur la posture d'observateur. Avant d'aborder la troisième partie, intéressons-nous aux trajets scolaires des enfants.

\section{DES CHEMINS OQUI RESTENT À DÉFRICHER}

\section{Venir à l'école : transport scolaire et adresses}

Il suffit de se promener dans les villes et les villages de Guyane aux heures d'entrée et de sortie scolaire pour comprendre que la question des transports scolaires est un enjeu dans l'accès à la scolarisation. En effet, le déficit et le coût des transports scolaires, souvent relayés dans la presse locale font également partie de nombreux rapports. Arrêtons-nous sur le rapport de la commission nationale consultative des droits de l'homme portant sur l'effectivité du droit à l'éducation dans les outremers. Regard particulier sur la Guyane et Mayotte, réalisé en 2017 :

" D'une part, le coût de l'abonnement annuel (110-120 euros par enfant) constitue une somme importante pour les familles modestes, particulièrement lorsqu'elles sont constituées de plusieurs enfants scolarisés [...]. D’autre part, en Guyane, l'accès à l'école pour les enfants du fleuve est conditionné par l'affectation de la commune au réseau de transport et par le passage du piroguier, qui peut s'avérer quelque peu aléatoire, et du nombre de places dans les embarcations (insuffisant notamment à Taluen et à Saint-Georges). " (CNCDH, 2017, p. 19).

Dans le bourg de la commune, une seule ligne de pirogue publique est effective. Elle relie l'embarcadère à un village nommé par ses habitants "le village Machine ". II s'agit d'un quartier situé à la sortie du bourg, à côté de la caserne militaire. La pirogue transporte quotidiennement une quinzaine d'enfants surtout inscrits en classe de maternelle. À côté de cet unique transport public, il existe un transport privé, souvent onéreux pour les familles. Un simple aller en taxi collectif coûte entre deux et cinq euros par personne. Ainsi, seuls les enfants les plus jeunes en bénéficient. Les autres traversent le village à pied. En fonction du lieu d'habitation, certaines familles investissent également dans des pirogues privées afin d'envoyer leurs enfants à l'école. Dans le groupe des élèves de I'UPE2A, une petite fille que nous nommerons Vanessa vit dans un village éloigné du côté français, non relié au réseau de transport public. Les familles du village se sont cotisées pour acheter une pirogue et de l'essence. Or, en relevant ses absences sur le cahier d'appel, nous constatons qu'elle ne fréquente pas sa classe tous les jours. Certaines fois, elle se justifie par un manque d'argent, d'autre fois pour cause de maladie. À travers I'expérimentation menée en 2016-2017, nous apprenons qu'elle attend tous les jours la pause méridienne du collège, pour rentrer chez elle avec la pirogue. Afin d'éviter trop de déplacements, un passage aller et un retour sont organisés pour tous les enfants de ce village. Lors de nos échanges, nous tentons de comprendre qui vient la chercher, mais cet enfant de CE2 n'a su nous répondre tant la question lui semblait étrange. Vanessa ignore à quelle heure précisément passe la pirogue et même qui la conduira. 
II existe également un autre chemin emprunté par les enfants, mais celui-là fait l'objet de silences et de non-dits puisqu'il nous conduit de l'autre côté du fleuve, " en face ", dans un autre pays: le Suriname. Contrairement à l'adresse indiquée dans son dossier scolaire, Lucas ${ }^{16}$ explique timidement qu'il vit dans un village situé "de l'autre côté de l'eau». Le fait d'y habiter est vécu comme un secret par bon nombre d'enfants et de jeunes, qu'il apparaît nécessaire de cacher. Dans les villages situés en face du bourg, nous retrouvons majoritairement des familles brésiliennes travaillant dans l'activité aurifère. Ces dernières sont souvent très mobiles en fonction des possibilités d'emploi qu'offrent les camps d'orpaillage illégaux. Ainsi, rares sont les enfants de ce côté-là du fleuve à suivre une scolarité, qui plus est continue. Depuis ces villages informels, il existe plusieurs chemins pour aller à l'école: marcher depuis chez soi jusqu'aux commerçants qui affrètent des pirogues pour les clients et ainsi profiter d'une traversée, avoir une pirogue privée ou payer un piroguier. Dans le village de Ronaldo, la marche n'est pas plébiscitée par les familles. Pour ces dernières, "le village n'est pas bon pour les enfants". En pleine expansion depuis 2016, ce village est une base arrière de l'orpaillage, rassemblant des commerces, des bars et des discothèques. De plus, le chemin séparant le village des commerçants se matérialise par un layon tracé entre le fleuve et des plantations. À certaines périodes de l'année et notamment en saison des pluies, il devient impraticable ou s'éloigne du fleuve pour entrer dans la forêt. Ainsi, les familles préfèrent faire appel au service d'une pirogue privée. Dans ce cas, le tarif du transport est aligné sur celui de la France: cinq euros l'aller. C'est le prix que paye Paola, la mère de Lucas pour l'envoyer à l'école depuis qu'elle s'est installée de ce côté du fleuve avec ses deux enfants.

Si l'enfant n'a pas à justifier d'une existence administrative française ${ }^{17}$ pour aller à l'école, il doit en revanche attester du fait qu'il relève d'un secteur scolaire ${ }^{18}$. En contradiction avec I'article L.131-5 du code de l'éducation, certaines mairies de Guyane exigent une facture d'eau, d'électricité ou bien un bail alors que nombre de familles vivent en habitat auto-construit. Ainsi le justificatif de domicile, qu'il soit titre de propriété, contrat de location ou attestation d'hébergement devient un document incontournable pour franchir le portail de l'école et ainsi bénéficier du droit à l'instruction. Cette dérive entraîne des stratégies de contournement visant à se procurer une adresse fictive, soit auprès d'un proche, soit auprès d'un tiers moyennant parfois une contrepartie financière. Selon les membres d'une association d'accompagnement dans l'accès aux droits rencontrés à Maripasoula, une domiciliation chez un tiers se monnayerait à hauteur de 400 euros. Parmi la quarantaine de dossiers de collégiens scolarisés et intégrés à I'UPE2A, rares sont ceux qui bénéficient d'une domiciliation relevant d'une association. Pour l'écrasante majorité, les enfants avaient dans leurs dossiers des attestations d'hébergement

16. Les prénoms ont été modifiés.

17. Voir notamment l'article L113-1 du code de l'éducation, la circulaire du ministère de l'Éducation nationale du 6 juin 1991 et la circulaire $n^{\circ}$ 2002-063 du 20 mars 2002 relative aux modalités d'inscription et de scolarisation des élèves de nationalité étrangère du premier et second degré.

18. Voir notamment l'article L312-10 du code de l'éducation sur la carte scolaire. 


\section{POLITIOQUES INCLUSIVES D’ÉDUCATION}

chez un tiers. Ces propos sont à mettre en corrélation avec le rapport du défenseur des droits sur l'accès aux droits et aux services publics en Guyane de 2016 :

"L'accès à l'information s'opère, comme sur un marché, selon un prix, celui du déplacement, de la traduction, de l'écrivain public, du ticket de passage pour une place favorable dans la file d'attente. Le recours à des intermédiaires communautaires, illégaux et payants, génère des trafics en tout genre (fraude documentaire, vol de courriers, malversation, racket). " (Mathieu, 2016, p. 33).

D'autre part, en consultant les dossiers et en discutant avec les enfants, nous constatons aussi que les familles sont très mobiles et déménagent souvent. Les équipes éducatives soulèvent la problématique des enfants qui disparaissent, quittant l'établissement d'une année sur l'autre ou en milieu d'année. Selon le principal adjoint du collège de Maripasoula, le départ brutal d'une famille pourrait s'expliquer par des raisons économiques, notamment suite à la fin d'un chantier d'orpaillage ou suite à des faits de violence. Néanmoins, ces départs soudains ne sont pas propres à la commune et de nombreux professionnels de l'éducation s'accordent sur I'hypermobilité des enfants en Guyane française.

"On le voit sur le second degré, entre le moment où les enfants passent le test [du Casnav], s'inscrivent et le moment où ils sont affectés dans une classe, ils ne sont plus à l'adresse qu'ils nous avaient indiquée au moment du test, voire six mois après. Ils ont changé d'adresse, donc, ils ne reçoivent pas leur notification. Je pense qu'il y a une forte mobilité des populations. Enfin, c'est une intuition, mais à mon avis, ça bouge beaucoup. " (Conseiller pédagogique Casnav, 2018).

Les professionnels rencontrés insistent sur la mobilité des enfants migrants ou étrangers comme cela est le cas du conseiller pédagogique du Casnav. Or, comme nous l'avons souligné en introduction, tous les enfants inscrits en UPE2A ne sont ni migrants ni étrangers en Guyane française. Cependant, ils ont tous dans leur dossier scolaire plusieurs adresses, soit dans la commune ou dans les communes voisines. En effet, il a été relevé jusqu'à cinq adresses différentes en fonction des justificatifs ${ }^{19}$ demandés dans le dossier des élèves inscrits en UPE2A au second degré. Ainsi, la mobilité semble concerner tous les enfants indépendamment de leur lieu de naissance ou de leur appartenance communautaire.

Dans son article Pratiques des lieux, modes d'habiter, régimes d'habiter: pour une analyse trialogique des dimensions spatiales des sociétés humaines, publié en 2006, Mathis Stock souligne un fait intéressant. L'accroissement de la mobilité ainsi que la différenciation des rythmes de déplacements auraient comme suite un changement de signification des lieux géographiques que les personnes pratiquent, transformant ainsi la représentation de l'habiter. On assisterait à une recomposition des valeurs assignées aux lieux, ce qui modifierait radicalement la manière dont les individus les

19. Extrait d'acte de naissance, justificatif de domicile, assurance scolaire, adresse des représentants légaux etc. 
habitent. Nous pouvons prendre l'exemple de cette petite fille qui nous a conduits sur le lieu de travail de sa mère plutôt qu'à son domicile. Dans la représentation de sa famille, elle avait indiqué vivre avec son père et sa belle mère. En discutant avec un autre enfant, nous comprenons qu'il possède trois lieux de vie dans le bourg, chacun correspondant à une activité et à un temps donné. La multiplicité des adresses traduit à la fois une forte mobilité des populations, caractéristique de I'histoire et de la géographie du territoire, mais également une grande précarité des familles. En insistant sur le caractère pluriel de la géographie des hommes, Mathis Stock nous invite également à penser les stratégies d'appropriation des lieux dans le cas d'habitat vécu comme temporaire ou transitoire. Dès lors: " [La] variable discriminante pour déterminer la familiarité d'avec les lieux n'est plus la distance, mais la fréquence de la pratique " (Stock, 2006, p. 7).

En passant le portail, en sortant de l'école, nous avons pu suivre ces élèves, ces enfants, traverser la frontière du monde scolaire pour rejoindre leur autre quotidien, celui de la maison.

\section{Vivre en territoire frontière : mobilité et précarité}

Quand nous avons rencontré Lucas en janvier 2017, il habitait dans le village de Ronaldo au Suriname. À ce moment-là, sa mère qui était partie sur un site d'orpaillage avec sa petite sœur l'avait confié à une amie. À la rentrée scolaire 2017, Lucas pourtant inscrit à l'école n'était pas présent. II est arrivé en janvier 2018 après avoir passé trois mois sur un site d'orpaillage avec sa mère et sa sœur. Lucas était alors installé dans une petite maison du bourg de Maripasoula. Bien que la maison soit au goût de Paola, ils sont repartis vivre du côté du Suriname en avril 2018. Paola y a finalement acheté une maison. La nouvelle maison comprend trois pièces et deux terrasses. Elle est toute en bois, sans eau ni électricité. Elle a coûté 263 grammes d'or, selon ses dires. Elle se situe au début du village et donne directement sur le fleuve. Elle est bien plus grande que les deux précédentes et toujours arrangée avec beaucoup soin. Paola travaillant dans l'orpaillage, cette maison lui permet de maintenir son activité - notamment pour stocker des denrées alimentaires à envoyer sur les camps - tout en donnant à ses enfants un espace pour vivre. Cependant, selon ses propos, les enfants ne sortent pas seuls au village, car il n'est pas adapté pour eux. Le village serait trop violent, trop dangereux. Elle le justifie par la forte présence de bars et de la prostitution. Quand Lucas souhaite faire du vélo, sa mère appelle une pirogue et paye cinq euros pour aller en face, sur la place de Maripasoula. Aujourd'hui, Paola, en situation irrégulière, souhaite quitter Maripasoula et Ronaldo pour s'installer avec ses enfants à Saint-Laurent-du-Maroni. Elle considère que la vie y sera plus facile pour ses enfants et la scolarité de meilleure qualité.

"Habiter temporairement les lieux géographiques semble faire de ces derniers de plus en plus des "lieux de projet" pour lesquels l'ancrage et l'appropriation symbolique de la part des individus sont éphémères, toujours sujets à négociation en fonction des situations et du moment dans la vie des individus. " (Stock, 2006, p. 11). 


\section{POLITIOQUES INCLUSIVES D’ÉDUCATION}

La difficulté des situations vécues notamment par les familles migrantes rappelle à quel point I'habitat constitue le pilier essentiel d'une projection dans la vie sociale du territoire et ceci quand bien même cet habitat se vit et se révèle précaire, peu confortable, voire informel. De ce point de vue, que I'on soit au bourg de Maripasoula ou au Suriname, les conditions de vie se ressemblent dans l'insalubrité. Manque d'accès à l'eau, à l'électricité, à quoi se rajoute un ramassage des ordures largement défaillant d'un point de vue logistique. Des études sur le foncier de deux quartiers de Maripasoula en attestent, comme différents échanges avec du personnel communal. À titre informatif, les maisons de fortune ${ }^{20}$ représentaient à Maripasoula en 2014, 31,1 \% des résidences principales contre 2,9 \% à Cayenne un an plus tard. Toujours selon I'Insee ${ }^{21}$, en 2014, 37,8 \% des résidences principales déclaraient avoir une douche ou une baignoire et des WC à l'intérieur du logement en 2014 à Maripasoula contre 91,9 \% à Cayenne en 2015. Enfin, seulement 3,2 \% des résidences principales étaient reliées au tout à l'égout contre 53,1 \% à Cayenne. Sans accès à l'eau potable dans le logement, la vaisselle familiale ou la douche se prend au fleuve. Ainsi, il n'est pas rare, très tôt le matin, de voir des enfants faire leur toilette au bord de l'eau, les ainés préparant une partie de la fratrie. Questionner l'expérience scolaire demande indubitablement de prendre en compte la question du transport et des conditions de vie des enfants.

" On doit bien se représenter que pour certains enfants, aller à l'école suppose de se lever très tôt de faire plusieurs heures de pirogue par jour, dans des conditions difficiles et aléatoires. Pour d'autres collégiens, cela signifie s'exiler dans une localité à 2 ou 3 jours de pirogue de sa famille, dans un milieu nouveau, soit en internat (souvent fermé le week-end), soit dans une famille d'accueil, peu formée ou motivée. " (Mathieu, 2016, p. 16).

Les chemins présentés par les enfants ont mis en lumière la détermination de certains parents à envoyer les enfants à l'école, à l'instar de Paola, achetant une pirogue, payant des transports privés ou monnayant une adresse du côté français. Ces expériences d'interactions multiples construites le long des parcours, des installations, des déménagements, des transactions pour survivre, interrogent et transforment les rapports entre l'école et la famille et contribuent à la redéfinition de la notion d'intégration (Missaoui, 2007, p. 387). Nous constatons que des stratégies familiales se mettent en place pour permettre la scolarisation des enfants. D'autre part, les expérimentations ont démontré la présence des parents au domicile. Dès lors, il nous faut interroger les relations famille-école et la place de la formation des enseignants dans ce territoire frontière d'Amazonie.

20. Source Insee. Pour Cayenne: RP2015 exploitation principale, géographie au 1er janvier 2017. Pour

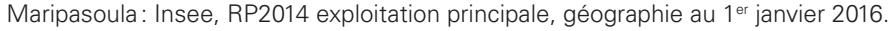

21. Source Insee, op. cit. 


\section{APPORTS DE LA RECHERCHE (EN ÉDUCATION) À LA FORMATION PÉDAGOGIOUUE}

\section{Enseignants et familles de part et d'autre du chemin}

Bien que les familles ne se rendent pas jusqu'au portail de l'école, elles attendent leurs enfants sur le pas de la maison. Cette apparente absence des parents dans le cadre scolaire souvent décrite par les professionnels de l'éducation cache surtout une forte distance entre le monde enseignant et familial (Lange, Pilon, 2012). Plusieurs éléments sont à prendre en compte dans cette approche distanciée des deux univers. D'une part, le français est loin d'être la langue la plus usitée à Maripasoula. La langue de socialisation, parlée par bon nombre d'habitants quelle que soit la communauté d'appartenance est le neenge tongo, dans sa variante Aluku. Elle est notamment parlée par les Brésiliens travaillant dans l'or, les commerçants asiatiques situés au Suriname, mais également par toute la population noire-marron. Ainsi pouvons-nous nous demander qui est allophone sur ce territoire: les enfants et les familles qui ne maîtrisent pas le français ou les enseignants qui ne parlent pas le nenge tongo? De plus, rares sont les enseignants issus des communautés du fleuve, noires-marrons ou amérindiennes. Les professionnels de l'éducation sont majoritairement créoles et métropolitains et $n^{\prime}$ ont souvent aucune attache personnelle avec le territoire. D'autre part, avec un chômage ${ }^{22}$ de plus de 70 \% à Maripasoula, les enseignants appartiennent à la catégorie socioprofessionnelle la plus élevée aux côtés des médecins. Ces professionnels souvent jeunes se heurtent à des difficultés d'adaptation. Isolés - cette commune est uniquement accessible par avion ${ }^{23}$ ou en pirogue ${ }^{24}$ - ils ont du mal à s'adapter à un mode de vie plus rural ainsi qu'à s'intégrer au paysage socioculturel. Dans les villages de l'intérieur et des fleuves, communément appelés par l'Éducation nationale " sites isolés ${ }^{25}$ ", les conditions de vie sont précaires pour les enseignants affectés, comme le souligne le rapport du Défenseur des droits:

"Les écoles implantées le long des fleuves ne sont accessibles qu'en pirogue et les conditions de vie précaire dues à l'isolement, au climat et à l'habitat ne facilitent pas la tâche d'enseignants en lien avec une population en majorité non francophone. Le plus difficile est de stabiliser les enseignants titulaires, en raison de mauvaises conditions de vie et de logements inadaptés, voire inexistants. » (Mathieu, 2016, p. 15).

De plus, l'institution scolaire est relativement récente sur les fleuves de Guyane. Au moment de la colonisation, l'école ne concernait que les enfants de colons blancs.

22. Le taux de chômage avoisinait les 74,1 \% en 2014 à Maripasoula contre 50 \% à Saint-Laurent du Maroni la même année et $29,4 \%$ à Cayenne en 2015, source Insee, op. cit.

23. Environ 170 euros, $A / R$, entre Maripasoula et Cayenne ou Saint-Laurent du Maroni.

24. Environ 50 euros et plus de 5 heures de pirogue jusqu'à Saint-Laurent du Maroni.

25. Les sites isolés correspondent, pour le rectorat de Guyane, à un certain nombre d'écoles qui ne sont pas accessibles par route et ne bénéficient pas de conditions optimales de communication (accès à Internet et téléphone). Ainsi qualifiés, ces établissements scolaires ouvrent droit à une indemnité financière, décret $n^{\circ}$ 77-1364 du 5 décembre 1977 portant attribution d'une indemnité en faveur des personnels relevant du ministre chargé de l'Éducation nationale en service dans certains postes isolés du département de la Guyane. 


\section{POLITIOQUES INCLUSIVES D'ÉDUCATION}

Après l'abolition de l'esclavage en 1848 et l'instauration de la laïcisation en 1888, l'école intégra peu à peu les enfants d'esclaves libérés. Cependant, les populations amérindiennes et marronnes situées sur les fleuves ne bénéficièrent pas de ces changements. Cette exclusion était renforcée par le statut du territoire autonome de I'Inini ${ }^{26}$ qui, entre autres, ne les soumettait pas à l'obligation scolaire.

"Ce découpage du territoire entérine la distinction démographique et politique entre, d'une part, les populations créole et métropolitaine dont les membres sont des nationaux et des citoyens français et, d'autre part, celles des Noirs marrons et des Amérindiens qualifiées de primitives et de tribales; "peuples indépendants" dont les membres, pour la très grande majorité, n'ont ni état civil ni, en conséquence, nationalité et citoyenneté française. " (Benoît, 2016, p. 241).

C'est à partir de la politique de francisation des années soixante que commença la scolarisation des enfants de ces populations. La fin du régime de I'Inini en 1968, la création des communes de l'intérieur, la dynamique démographique du département et la volonté de dispenser une éducation à l'ensemble des enfants sont autant de facteurs qui ont permis la création d'écoles publiques dans ces communes (Puren, 2014). Ainsi, à Maripasoula, la première école primaire du bourg, du nom du préfet qui a encouragé le développement des communes de l'intérieur, a été ouverte en 1968, suivie de l'ouverture de l'école de Twenké en 1972. II a fallu attendre 1984 pour voir une école publique s'établir à Antecume Pata et en 1998 à Pidima, dernier poste scolaire sur le fleuve Maroni.

Le caractère récent de l'institution scolaire et les fortes distances entre l'univers familial et scolaire influencent la manière dont les familles vont investir le lieu de l'école. Certains parents n'y trouvent pas leur place ou estiment qu'ils n'ont rien à y faire. De l'autre côté, les enseignants émettent des réserves quant au fait de sortir des murs de l'école pour rencontrer les familles. Nous l'avons vu lors de la présentation des premières expérimentations. Nombreux ont été les enseignants rencontrés à interroger les limites de leur mission. "Jusqu'où puis-je aller en dehors de l'école? Que faire de l'histoire personnelle des élèves de ma classe? En quoi connaître ce vécu va-t-il m'aider dans le développement de compétences et favoriser les apprentissages de mes élèves? "Bien que la majorité des enseignants de l'école étudiée ait répondu, lors d'un questionnaire, qu'il était nécessaire de prendre en compte le contexte de vie pour mener les apprentissages, nous constatons que ces derniers restent démunis, en proie à leur propre représentation subjective du métier. Dès lors, nous nous devons d'interroger le travail en équipe et la formation continue sur ce territoire isolé.

26. Le territoire autonome de I'Inini a été créé par le décret du 6 juin 1930. II s'agissait d'une unité administrative gérée directement par l'État avec à sa tête un gouverneur. Le territoire devint arrondissement de l'Inini en 1951 avec les mêmes dispositions. 


\section{Former en territoires isolés}

Alors que la question de la formation des professeurs des écoles s'invite de nouveau dans les débats ministériels, la commission nationale consultative des droits de I'homme pointe le manque d'accompagnement des personnels en Guyane française (CNCDH, 2017, p. 27). Le manque de professionnels éducatifs ${ }^{27}$ et de personnels formés sont deux grandes problématiques scolaires des territoires de Guyane. Selon les propos du Dasen ${ }^{28}$ de l'académie de Guyane, Joseph Vallano, auditionné par la commission nationale consultative des droits de l'homme, "pour le premier degré, le rectorat de Guyane constate, en moyenne, une demande d'entrée de titulaires pour dix demandes de sorties du territoire ". Ainsi l'académie doit recourir massivement au recrutement de contractuels. À titre d'exemple, ces derniers représentaient à la rentrée 2015 près de 33,4 \% des effectifs en Guyane pour le second degré alors que la moyenne nationale est de 7,5\%. Les UPE2A souffriraient du même manque de professionnels que les classes ordinaires. Selon I'IA-IPR ${ }^{29}$ de lettres de I'académie, parmi les dix-neuf professeurs d'UPE2A du second degré de l'année 2017-2018, la moitié serait titulaire et l'autre contractuelle.

" [...] En formation continue, y a peu de formation et puis y a un gros turn-over d'enseignants, même dans les UPE2A. On a des contractuels, on a des gens qui tournent, faut les former tous les ans, donc c'est pas simple. " (Conseiller pédagogique Casnav, 2018).

À Maripasoula, l'enseignante de I'UPE2A de l'école élémentaire est une professeure des écoles titulaire, travaillant dans la commune depuis quinze ans. Elle fait partie des quelques personnels qui sont restés plus de trois ou quatre ans sur ce territoire enclavé. Suite au départ de la professeure de I'UPE2A, il y a cinq ans, I'inspecteur de circonscription lui a proposé de prendre le poste. Depuis, elle exerce dans I'UPE2A, sans spécialisation particulière.

"Alors comment j'ai été recrutée? En fait j'étais déjà à l'école depuis 4/5 ans avec une classe de CE1 et CE2, etc., et un jour la fille qui avait la Clin et qui est restée 5 ans ici a demandé sa mutation sur Cayenne et l'inspecteur, notre inspecteur de circonscription, m'a proposé par l'intermédiaire de mon directeur d'intégrer cette classe [...]. Mon directeur était dans ses petits souliers et ne savait pas trop comment me présenter cela, car quelquefois je suis un peu [soupirs] pour ce qui est de la nouveauté, etc. Je pense qu'il faut quand même réfléchir, étudier un peu. Mais là l'inspecteur m'a demandé de réfléchir en 1 heure. En fait c'est le directeur qui l'avait eu au téléphone et qui lui m'a laissé réfléchir en pleine classe et qui m'a dit dans 1 heure à la récréation tu viens me voir et tu me dis oui ou non. Voilà. Donc j'étais avec ma classe et dans ma tête il fallait que je réfléchisse vite et je me suis dit "Allez, fonce."

27. Personnels de l'Éducation nationale enseignants et non enseignants.

28. Dasen: Directeur académique des services de l'Éducation nationale.

29. IA-IPR: Inspecteur d'académie - Inspecteur pédagogique régional. 


\section{POLITIOQUES INCLUSIVES D’ÉDUCATION}

Comme c'était à une époque où avoir une classe complète devenait compliqué pour moi à cause de la discipline, du bruit, de tout ça, j'étais fatiguée et avais des difficultés, je me suis dit qu'il fallait passer à autre chose. " (Enseignante en UPE2A, 2017).

Le renouvellement important des équipes et le recours massif à un personnel contractuel ont une incidence sur la qualité de l'enseignement scolaire. Ces équipes peu ou pas expérimentées, souffrent également d'un manque de formation continue.

" La CNCDH a été alertée à plusieurs reprises sur la difficulté d'accéder à la formation continue en Guyane et à Mayotte, malgré l'effort des services du rectorat pour enrichir le plan de formation continue (pédagogie différenciée, apprentissage en contexte plurilingue...). Le recrutement difficile de formateurs spécialisés constitue un premier obstacle. Les vives difficultés que rencontrent certains enseignants pour suivre une formation, en raison de l'éloignement du lieu d'affectation des centres de formation et, le cas échéant, du nombre insuffisant de personnels remplaçants, en constitue un second, notamment mis en exergue en Guyane. " (CNCDH, 2017, p. 28).

Les formations ont majoritairement lieu à Cayenne ou à Saint-Laurent-du-Maroni. Cette professeure de I'UP2EA de l'école élémentaire qui a suivi deux formations en cinq ans, I'une à Cayenne et l'autre à Saint-Laurent-du-Maroni, met en avant sa lassitude face aux déplacements.

"L'année dernière et celle d'avant, j'ai pas eu de proposition de stage. Je suppose qu'il faut de l'argent pour cela. Et puis, même si c'est un détail, quand tu vois que tu n'es remboursée ou pas remboursée sur les frais et que ça peut prendre longtemps, d'ailleurs mon truc à Cayenne je ne sais même plus si cela m'a été remboursé, et bien ça participe au fait que tu souhaites pas forcément participer. » (Enseignante UPE2A, 2017).

Seules les formations de circonscription du premier degré ont lieu sur place. Des échanges informels avec des professeurs ont révélé le manque d'adéquation entre les formations et leur quotidien professionnel. Il est intéressant de noter que très peu de formations sont proposées autour des questions d'allophonie ou de plurilinguisme alors que chaque enseignant y est confronté dans sa pratique quotidiennement. Ainsi, la professeure de I'UPE2A, à l'instar d'autres, regrettait que le Casnav ne se soit pas déplacé à Maripasoula avant l'expérimentation présentée dans cet article.

"Mais c'est vrai qu'on est peu sollicité... on a été peu sollicité sur les fleuves ces trois dernières années. Normalement y a deux possibilités, soit c'est eux qui nous sollicitent, soit c'est nous qui proposons des formations à mettre dans leur plan de formations, et si les formations sont acceptées et mises dans leur PAF [Plan académique de formation], à ce moment-là, on se déplace. Mais 
on attend effectivement que les propositions viennent des circonscriptions. " (Conseiller pédagogique Casnav, 2018).

Malgré des difficultés apparentes, des efforts sont à noter. D'un point de vue de l'expérimentation proposée, l'enseignante a manifesté son intérêt et son enthousiasme:

" Je regrette qu'en 15 ans on n'ait pas eu ça. Dans les formations, on est à la table, on est des élèves. [...]. Je préfère faire une formation qui n'est pas une formation, directement dans ma classe où l'on parle et à l'extérieur. Je suis d'accord pour faire des trucs comme ça. " (Enseignante UPE2A, 2018).

Le conseiller pédagogique quant à lui s'inscrivait dans la volonté d'initier des pratiques innovantes en UPE2A et souhaitait développer plus de co-intervention directe de I'enseignante d'UPE2A dans les autres classes. L'idée de la boîte à histoires devait permettre ces échanges entre collègues. Ceci a eu lieu en CP avec une collègue. Sans le dire ainsi, ce premier travail de collaboration vient poser les bases d'une réflexion plus globale sur la recherche-action-formation et sur les formes de coopération possibles dans les territoires isolés de Guyane. À l'échelle de l'académie, la mise en place d'un dispositif alternant ${ }^{30}$ en formation initiale au sein l'Espé de Guyane depuis la rentrée 2015 ou le déploiement des UPE2A ${ }^{31}$ sur le territoire sont autant d'éléments à prendre en compte.

\section{CONCLUSION}

À travers cet article, nous avons pris le parti d'interroger l'expérience scolaire des enfants en sortant de l'école. En effet, les trajets et les conditions de vie des familles sont apparus comme des éléments essentiels à la compréhension des pratiques scolaires. Comment traduire les absences régulières de Vanessa si nous ne savons pas que son transport dépend d'une pirogue privée et de l'achat de l'essence? Comment comprendre les absences longues de Lucas sans prendre en compte la profession de sa mère? Loin de justifier des pratiques, ces éléments biographiques permettent d'appréhender l'enfant dans son intégralité et pas uniquement l'élève dans la classe. À travers les différentes expérimentations de recherche, conduites sur deux années scolaires, les enfants ont pu mettre en mots et en image leur trajet quotidien, leur famille et leurs lieux d'habitation. Plusieurs constats ont été présentés dans cet article. D'une part, dans cet espace frontière, nous avons remarqué une forte mobilité des familles qu'elles soient nées en Guyane ou à l'étranger. Cette mobilité peut être rythmée par les chantiers d'orpaillage illégaux, principale activité économique de la commune, où les enfants doivent alors investir le projet migratoire

30. Expérimenté en 2015/2016 dans les académies de Créteil et de Guyane, le dispositif permet dès le master 1 MEEF d'avoir un temps d'observation puis de pratique accompagnée et de responsabilité face aux élèves et une rémunération sur l'année financée par le rectorat, en même temps que son inscription étudiante. En contrepartie, l'étudiant s'engage à se présenter aux concours en fin de master 1.

31. À la rentrée 2018, l'académie comptait 19 UPE2A et 27 UPE2A-NSA dans les collèges et 62 dispositifs en école, (Projet académique 2018-2021, p. 4) 


\section{POLITIOQUES INCLUSIVES D’ÉDUCATION}

des familles. Rares sont les familles comme celle de Paola, qui, vivant au Suriname, décident d'envoyer leurs enfants à l'école, qui plus est française. La plupart des enfants de ce côté-là du fleuve ne sont pas scolarisés ou ne le sont plus, suivant les travailleurs de l'or de camp en camp. Cependant, les déplacements à Maripasoula ne concernent pas uniquement les familles migrantes. Entre les mobilités transfrontalières et intercommunales, les enfants noirs-marrons et amérindiens se déplacent ou sont envoyés chez un membre de la famille. Après un ou deux ans passés au Suriname ou dans la commune voisine, ils reviennent. Ils ont pu être scolarisés ou ne l'ont pas été. Enfin, nous avons pu noter la mobilité des personnels éducatifs. Mutés pour une première affectation, à la recherche d'une première expérience professionnelle ou encore en quête d'un ailleurs après plusieurs années en France métropolitaine, les professionnels s'inscrivent sur le territoire pour un temps donné se réduisant à quelques années. Enclavés dans le territoire et coupés du littoral, ces enseignants sont souvent démunis face aux enfants qu'ils accueillent dans leur classe. Accrochés à la posture subjectivée de l'élève, ils rencontrent des difficultés à prendre en compte l'enfant et son histoire. Ainsi, comme cela a été explicité dans l'article, nous avons souhaité réaliser une méthodologie de recherche ayant un volet pédagogique. Cette dernière permettait de développer les compétences des enfants, mais surtout d'interroger la formation continue des enseignants sur ce territoire, où, contrairement aux personnes, formations et bonnes pratiques ont dû mal à circuler.

\section{Références}

Alby, S. (2008). Faire faire et faire mieux dire à des élèves dans une école de l'Ouest guyanais. Le Français dans Le Monde, CLE International, 98-110.

Armagnague-Roucher, M., Cossée, C., Cossée-Cruz, E., Hieronimy, S., et Lallouette, N. (2017). Combiner sociologie et arts dans le recueil des données: Éléments pour une conceptualisation des méthodes artistiques dans les enquêtes qualitatives: l'exemple d'une recherche sur la scolarisation des enfants migrants. Migrations Société, 167(1), 63. https://doi.org/10.3917/migra.167.0063

Bassargette, D., et Di Meo, G. (2008). Les limites du modèle communal français en Guyane: le cas de Maripasoula. Les Cahiers d'Outre-Mer, 241-242, 49-80. https://doi.org/10.4000/com.3273

Benoît, C. (2016). Pampila et politique sur le Maroni: de l'état civil sur un fleuve frontière. Histoire de la justice, 26, 237-259.

Commission nationale consultative des droits de l'homme. (2017). Avis sur l'effectivité du droit à l'éducation dans les outremers. Regard particulier sur la Guyane et Mayotte. Paris: France.

Dossier complet commune de Cayenne. (2018). [en ligne] consulté le 10 mai 2018, https://www.insee. fr/fr/statistiques/2011101?geo=COM-97302

Dossier complet commune de Maripasoula. (2018). [en ligne] consulté le 10 mai 2018. https://www.insee. fr/fr/statistiques/2011101?geo=COM-97353

Farmer, D. (2016). Migrations et "nouvelles mobilités » : regards d'élèves et d'enseignants dans une école de langue française en Ontario (Canada). Alterstice, 6(1), 105-120. 
Hubert, B. (2014). Faire de sa vie une fiction. Des migrations en écriture avec des enfants d'école élémentaire. Hommes et migrations. Revue française de référence sur les dynamiques migratoires, 1306, 23-30. <https://doi.org/10.4000/ hommesmigrations.2792>

Lange, M.-F., et Pilon, M. (2009). Introduction: L'inégale soumission des familles aux impératifs scolaires. Cahiers de la recherche sur l'éducation et les savoirs, 8, 7-16.

Leglise, I., et Puren, L. (2005). Usages et représentations linguistiques en milieu scolaire guyanais. Univers Créoles, écoles Ultramarines, 5, 67-90.

Léglise, I. (2008). Plurilinguisme et migrations en Guyane française. Migrations et plurilinguisme, 94(100). Consulté à l'adresse < http://halshs.archives-ouvertes. $\mathrm{fr} / \mathrm{halshs}-00298611>$

Ligue des Droits de l'Homme, (2014). La carence institutionnelle dans l'établissement de l'état civil, vecteur d'atteintes à l'accès aux droits dans l'ouest guyanais. Rapport de mission du groupe de travail " outre-mer ». Paris: France.

Mathieu, Y. (2016). Accès aux droits et aux services publics en Guyane. Compte rendu du déplacement du Défenseur des droits en octobre 2016. Défenseur des droits.

Mairie de Maripasoula. (2013). Rapport de présentation du plan local d'urbanisme, commune de Maripasoula. Guyane française.

Missaoui, H.-S. (2007). L'usage intermittent de l'école: Des temporalités scolaires redéfinies par des enfants gitans et maghrébins nouveaux arrivants. Espace populations sociétés, 2-3, 385-396. https://doi.org/10.4000/eps.2255

Puren, L. (2014). Contribution à une histoire des politiques linguistiques éducatives mises en œuvre en Guyane française depuis le XIXe siècle. In I. Léglise et B. Migge (dir.), Pratiques et représentations linguistiques en Guyane: Regards croisés (pp. 279 -295). Montpellier: IRD Éditions. Consulté à l'adresse < http:// books.openedition.org/irdeditions/6957>

Stock, M. (2006 a). L'hypothèse de I'habiter poly-topique: pratiquer les lieux géographiques dans les sociétés à individus mobiles. Revue électronique des sciences humaines et sociales. Consulté à l'adresse <http://www.espacestemps.net/ articles/hypothese-habiter-polytopique/s

Stock, M. (2006 b). Pratiques des lieux, modes d'habiter, régimes d'habiter: pour une analyse trialogique des dimensions spatiales des sociétés humaines. Travaux de I'Institut de géographie de Reims, 115 -118, 213-230. 